\title{
Real time optical pressure sensing for tactile detection using gold nanocomposite material
}

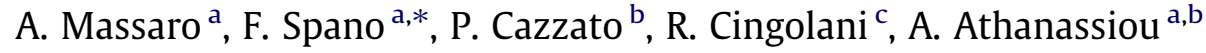 \\ a Italian Institute of Technology IIT, Center of Bio-Molecular Nanotechnology, Arnesano 73100, Italy \\ ${ }^{\mathrm{b}}$ National Nanotechnology Laboratory, Institute of Nanoscience of CNR, 73100 Lecce, Italy \\ ' Italian Institute of Technology IIT, Via Morego 30, 16163 Genova, Italy
}

\section{A R T I C L E I N F O}

\section{Article history:}

Available online 4 January 2011

\section{Keywords:}

Nanocomposite

Light coupling

Fiber pressure sensor

Tactile sensor

\section{Introduction}

Sensory information of human skin for feeling materials and determining many of their physical properties is provided by sensors in the skin. This tactile information is related to the sense of touch, one of the five senses including sight, hearing, smell, and taste. Presently, many researchers are attempting to apply the five senses to intelligent robot systems. In particular, many kinds of tactile sensors combining small force sensors have been introduced into intelligent robots. These tactile sensors, which are capable of detecting contact force, vibration, texture, and temperature, can be recognized as the next generation information collection system. Future applications of engineered tactile sensors include robotics in medicine for minimally invasive microsurgeries, military uses for dangerous and delicate tasks, and automation of industry. Some tactile sensors and small force sensors using microelectromechanical systems (MEMS) technology have been introduced. MEMS tactile sensing work has mainly focused on silicon-based sensors that use piezoresistive [1-3] or capacitive sensing [4-6]. These sensors have been realized with bulk and surface micromachining methods. Polymer-based devices that use piezoelectric polymer films [7-9] such as polyvinylidene

\footnotetext{
* Corresponding author.

E-mail addresses: alessandro.massaro@iit.it (A. Massaro), fabrizio.spano@iit.it (F. Spano).
}

fluoride (PVDF) for sensing have also been demonstrated. Although these sensors offer good spatial resolution due to the use of MEMS techniques, there remain some problems with respect to application to practical systems. In particular, devices that incorporate brittle sensing elements such as silicone based diaphragms or piezoresistors, including even those embedded in protective polymers, have not proven to be a reliable interface between a robotic manipulator and the manipulated object. Previous efforts have been hindered by rigid substrates, fragile sensing elements, and complex wiring. These drawbacks can be compensated by utilizing flexible optical fiber sensors and transducers. In addition, optical fiber sensors have immunity to electromagnetic fields, can be easily multiplexed and integrated with small led sources, by providing a good alternative for the implementation robotic tactile sensors [10]. Therefore, in this paper, we present a newly designed optical fiber force sensor based on electromagnetic (EM) coupling effect. The sensor is illustrated in Fig. 1a: a tapered multimode Si fiber couples the EM field coming from a broad band lamp source with the flexible gold/PDMS nanocomposite material (GNM) illustrated in Fig. 1b. PDMS polymer film was used since it is suitable for the generation of gold nanoparticles starting from gold precursors $[11,12]$. The formed gold nanoparticles increase the effective refractive index of the PDMS and support the EM coupling with the tapered region. A possible implementation of the proposed sensor in tactile finger robotic sensor configuration is showed in Fig. 1c and $d$ where a tapered fiber passes inside a PVC support representing finger shape. 

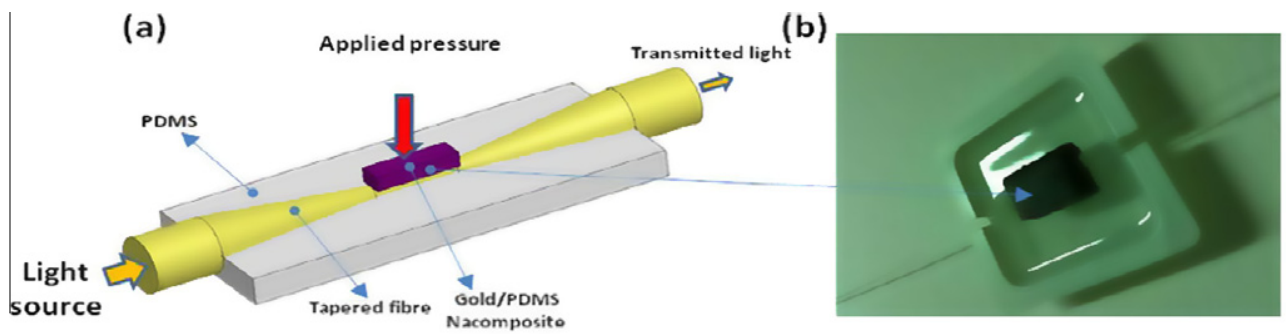

(c)

(d)
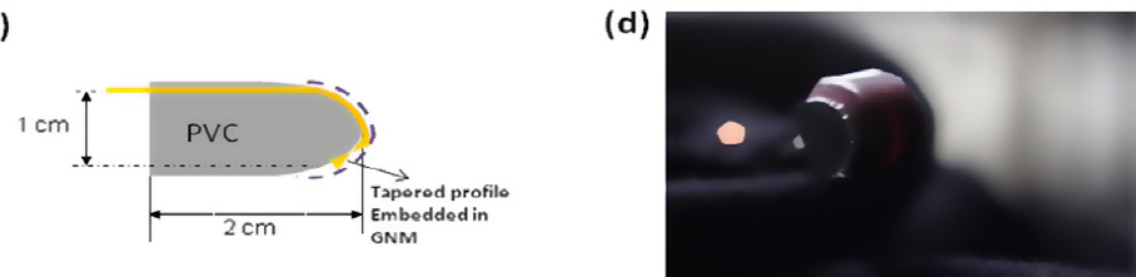

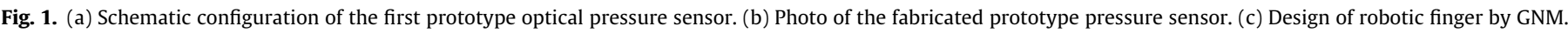
(d) Image of the light coupled inside the GNM of the robotic finger.

\section{Technology, basic principle, optical measurements, and discussions}

By applying different weights to the prototype of Fig. 1a that can be translated to pressure forces to the sensor, we observe, by means of an optical multichannel analyzer (OMA), in real time the intensity reduction of the transmittivity response at the output of the fiber. This effect is most likely due to displacement of gold nanoparticles near the tapered region during the pressure application. The GNM material, schematically reported in Fig. 2a, includes small gold particles with different dimensions as proved also by the Atomic Force Microscopy (AFM) images of the crosssection of the GNM shown in Fig. $2 b$ and $c$. The technological aspects and the pressure application modalities of the proposed sensors are illustrated in details in Fig. $2 \mathrm{~d}-\mathrm{g}$ : the first half of the tapered fiber (coated silica/core silica multimode FG-365-LER Thorlabs optical fiber) is embedded in PDMS material in order to improve a mechanical stability; the deposition of the PDMS (a)

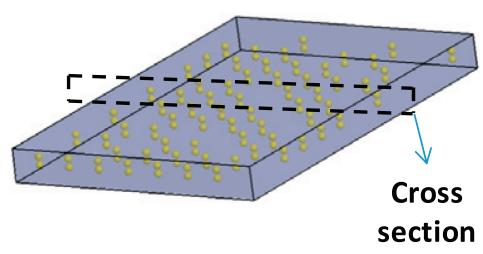

(d)

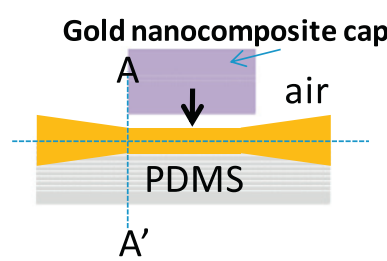

(b)

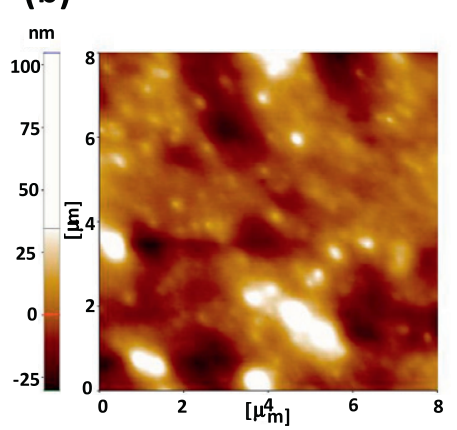

(e)

(f)

pressure

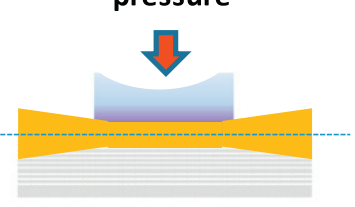

(c)

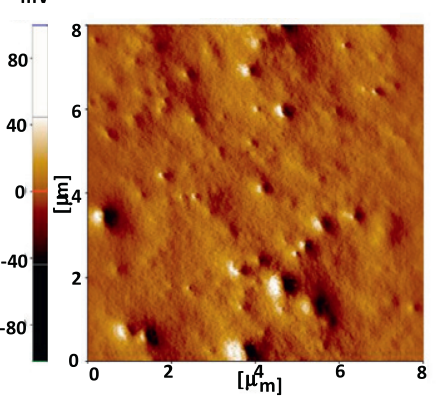

(g)
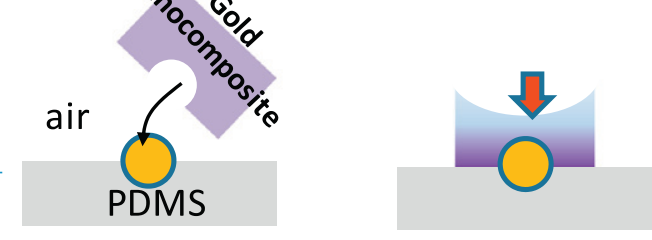

(h)

(i)

(I)
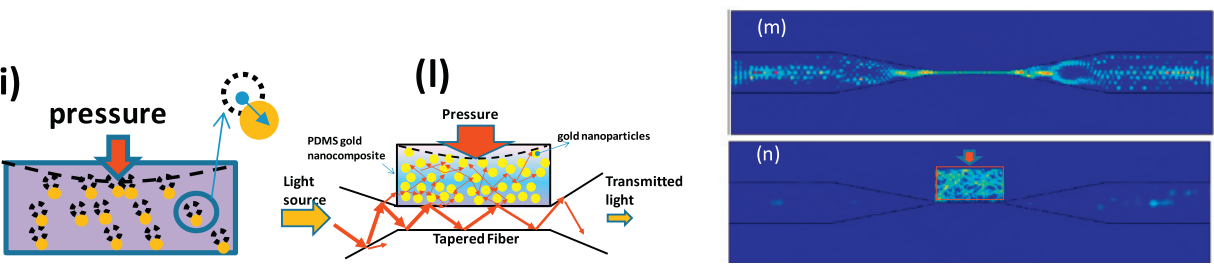

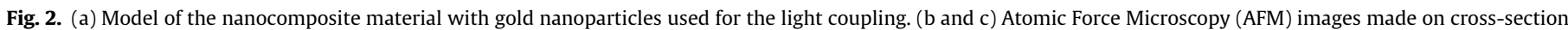

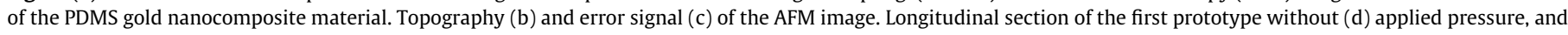

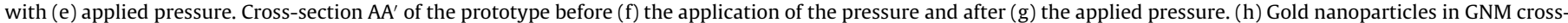

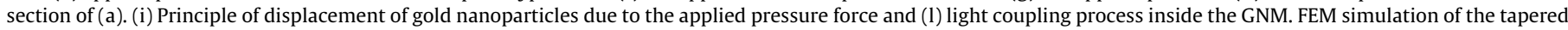
fiber and of the tapered fiber with (n) the GNM and after (m) the application of a force. 
(a)

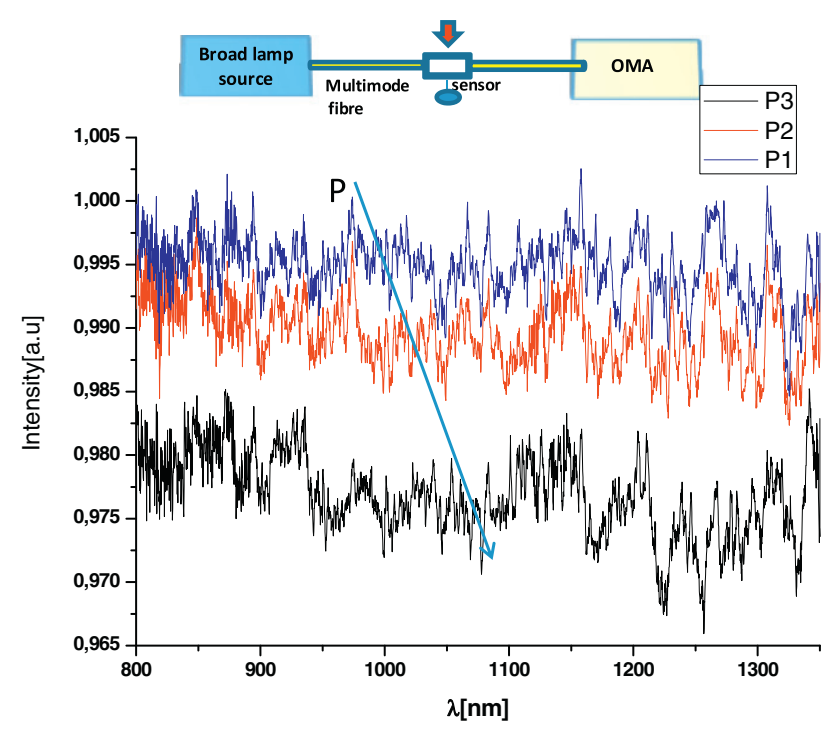

(b)
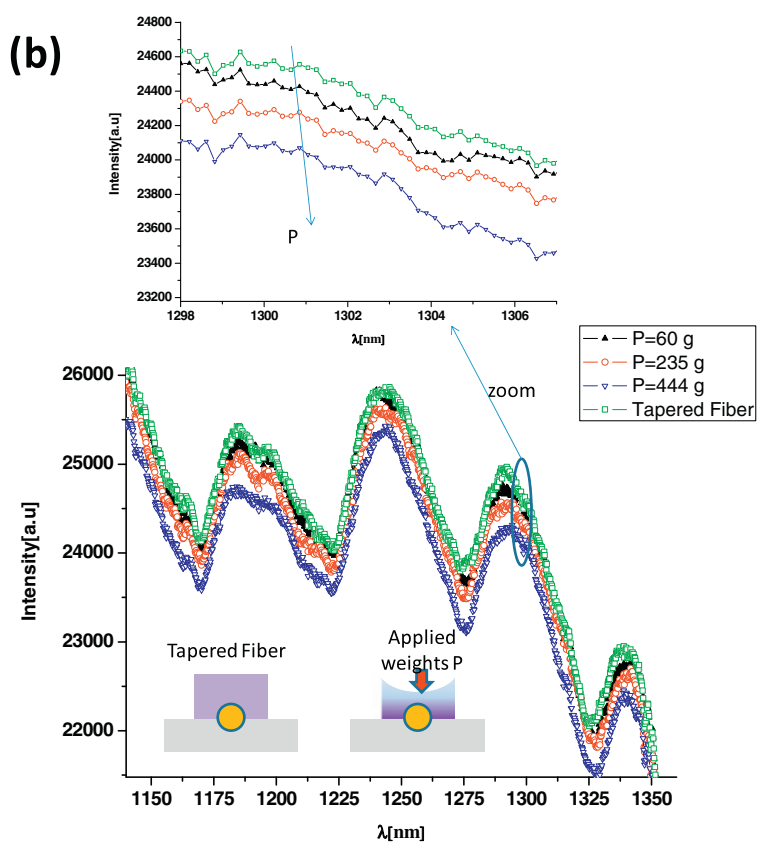

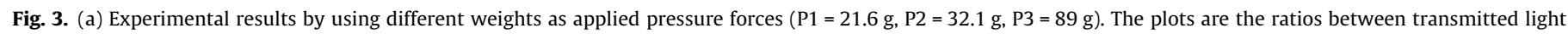

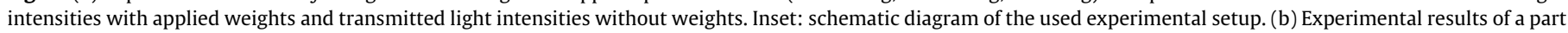
of the transmitted spectrum of the first prototype; inset above: zoomed image of a spectrum region; inset below: geometrical configurations in operating modes.

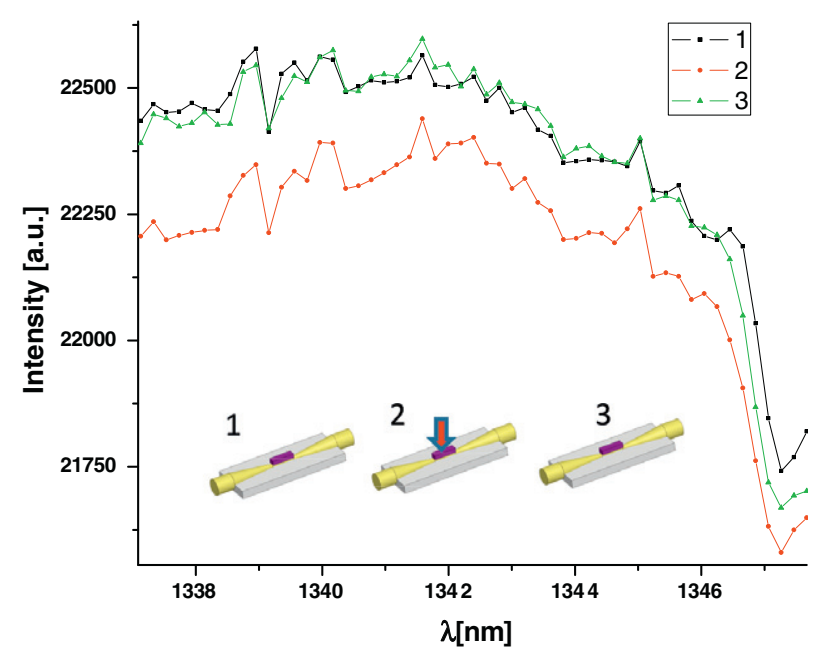

Fig. 4. Real time characterization of the pressure sensor by means of transmittivity spectrum: the plot 1 represents the initial step (no forces are applied), the plot 2 indicates the variation of the transmittivity response due to the applied weight of $118 \mathrm{~g}$, and plot 3 is the transmittivity response immediately $(0.6 \mathrm{~s}$ is the elastomeric time response) after the removing of the weight.

material (obtained by mixing a curing agent of $1: 10$ weight ratio to base polymer) is controlled by putting PDMS liquid droplets step by step in order to immerse only the first half of the tapered fibered as illustrated in all the sections of Fig. $2 \mathrm{~d}-\mathrm{g}$. About two days are necessary in order to perform the solid state of the PDMS in ambient temperature. Fig. $2 \mathrm{~d}$ and e show the longitudinal section of the sensor prototype without and with the applied pressure force, respectively: the GNM piece (PDMS material with gold nanoparticles) behaves as a cap and cover the region of the tapered fiber characterized by $1 \mathrm{~cm}$ of the total tapered profile length and about $5 \mathrm{~mm}$ of central core region without cladding. The cross-section of the first prototype is designed as shown in Fig. 2f where the GNM follows the shape of the fiber along the tapered region: this proce- dure allows to improve an equilibrium of the cap during the pressure application: as indicated in the cross-section of Fig. $2 \mathrm{~g}$, and according with the PDMS properties, the cap will improve an adherence with the tapered fiber during the pressing process. The physical basic principles of the sensors is based on the gold nanoparticles displacement near the GNM/fiber interface due to the applied force as schematically illustrated in Fig. $2 \mathrm{~h}$ and $\mathrm{i}$. The applied force improves a light scattering process (see Fig. 21) inside the GNM by decreasing the intensity of the light at the output of the fiber. The approximated finite element method (FEM) simulations of Fig. $2 \mathrm{~m}$ and $\mathrm{n}$ explains this principle by observing that the main EM power is coupled with the GNM after the application of a force. Due to the technological aspects, the proposed prototype of Fig. 1b is suitable with applied weight forces ranging from 10 to $400 \mathrm{~g}$. Applying weights more gold nanoparticles move near the coupling interface and the transmittivity shows an higher reduction of the intensity as illustrated in Fig. 3a where is reported also the scheme of the used experimental setup. Fig. 3a illustrates some measurements as the ratios between transmitted light intensities with applied weights and transmitted light intensities without weights. Besides, the whole optical spectra in a defined region are showed in Fig. 3b: also in this case a strong reduction of the optical transmitted intensity due to the applied weight forces are observed. The stable operating condition of the proposed sensor is proved by Fig. 4 where the optical response returns in the initial configuration after the measurements: high transmittivity response velocity of light is also checked by measuring an elastomeric time response of $0.6 \mathrm{~s}$. The measurement of the elastomeric sensor reactivity of $0.6 \mathrm{~s}$ (creep type measurements) is performed by TA Instruments DMA Q800 machine (dynamical mechanical analyzer).

\section{Conclusion}

We present in this work a new type of fiber sensor based on the optical coupling in PDMS gold nanocomposite material. The new optical approach is suitable for the implementation in robotic systems for applied weight forces ranging from 10 to $400 \mathrm{~g}$. High 
sensitive pressure sensor for the detection of few grams forces and electronic signal processing are under investigation.

\section{References}

[1] S.M. Firdaus, I.A. Azid, O. Sidek, K. Ibrahim, M. Hussien, Micro Nano Lett. 5 (2010) 85-90.

[2] B.J. Kane, M.R. Cutkosaky, T.A. Kovacs, J. Microelectromech. Syst. 9 (2000) 425434

[3] D.J. Beebe, A.S. Hseih, D.D. Denton, R.G. Radwin, Sens. Actuators A 50 (1995) 55-56.
[4] J. Il Lee, X. Huang, P.B. Chu, IEEE J. Microelectromech. Syst. 18 (2009) 660-670.

[5] B.L. Gray, R.S. Fearing, in: Proceedings of the IEEE International Conference on Robotics and Automation, 1996, pp. 1-6.

[6] M. Leineweber, G. Pelz, M. Schmoidt, H. Kappert, G. Zimmer, Sens. Actuators A 84 (2000) 236-245.

[7] C. Li, P.M. Wu, L.A. Shutter, R.K. Narayan, Appl. Phys. Lett. 96 (2010) 053502.

[8] E.S. Kolesar, C.S. Dyson, J. Microelectromech. Syst. 4 (1995) 87-96.

[9] R.R. Reston, E.S. Kolesar, in: Proceedings of the IEEE NAECON, 1990, pp. 11391144.

[10] J.S. Heo, J.H. Chung, J.J. Lee, Sens. Actuators A 126 (2006) 312-327.

[11] Q. Zhang, J.J. Xu, Y. Liu, H.Y. Cuen, Lab Chip 8 (2008) 352-357.

[12] C.E. Hoppe, C. Ridriguez-Abreu, M. Lazzari, M.A. Lopez-Quintela, C. Solans, Phys. Status Solidi A 205 (2008) 1455-1459. 